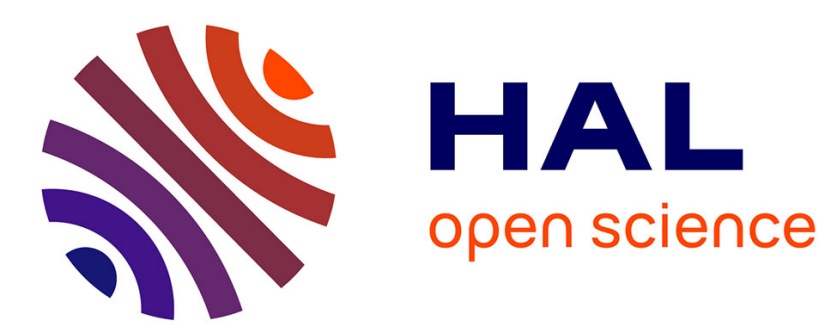

\title{
Energy efficiency comparison of Outphasing and IDP techniques with non-linear power amplifiers
}

\author{
Khaled Tahkoubit, Hmaied Shaiek, Daniel Roviras, Adda Ali-Pacha
}

\section{To cite this version:}

Khaled Tahkoubit, Hmaied Shaiek, Daniel Roviras, Adda Ali-Pacha. Energy efficiency comparison of Outphasing and IDP techniques with non-linear power amplifiers. 2020 3rd International Conference on Advanced Communication Technologies and Networking (CommNet), Sep 2020, Marrakech, France. pp.1-5, 10.1109/CommNet49926.2020.9199638 . hal-02953599

\section{HAL Id: hal-02953599 \\ https://hal.science/hal-02953599}

Submitted on 30 Sep 2020

HAL is a multi-disciplinary open access archive for the deposit and dissemination of scientific research documents, whether they are published or not. The documents may come from teaching and research institutions in France or abroad, or from public or private research centers.
L'archive ouverte pluridisciplinaire HAL, est destinée au dépôt et à la diffusion de documents scientifiques de niveau recherche, publiés ou non, émanant des établissements d'enseignement et de recherche français ou étrangers, des laboratoires publics ou privés. 


\title{
Energy efficiency comparison of Outphasing and IDP techniques with non-linear power amplifiers
}

\author{
Khaled Tahkoubit*, Hmaied Shaiek ${ }^{\dagger}$, Daniel Roviras ${ }^{\dagger}$, and Adda Ali-Pacha* \\ *LACOSI Laboratory, USTO-MB university \\ Po Box 505 El M'Naouer Oran 31000, Algeria \\ Email: khaled.tahkoubit, adda.alipacha@univ-usto.dz \\ CEDRIC/LAETITIA Laboratory, CNAM \\ 292 rue Saint Martin 75141 Paris, France \\ Email: hmaied.shaiek, daniel.roviras@cnam.fr
}

\begin{abstract}
OFDM is a prominent multicarrier modulation (MCM) waveforms for wireless systems. It has a very high Peak to Average Power Ratio (PAPR) due to the very great dynamic of the time domain signal. At high energy efficient level, the high PAPR is problematic, especially when non linear power amplifiers (PA) are used. PA linearization together with PAPR reduction techniques are two classical techniques for improving energy efficiency and BER performance. In this paper, two low PAPR techniques with similar transmitter architecture will be compared, in terms of energy efficiency and BER performance : Outphasing method which generates two emitted signals with constant envelope and Iterative Dichotomy PAPR Reduction (IDP) method which creates two emitted signals with low PAPR. In the two cases, the two generated signals are non-linearly amplified and further combined before the antenna device. Simulation results show that the IDP technique outperforms Outphasing technique in terms BER and emitted power.

Index Terms-OFDM, PAPR, PA, Outphasing, IDP
\end{abstract}

\section{INTRODUCTION}

Today's communication systems are increasingly demanding in terms of reliability, broadband transmission, mobility, spectral efficiency and energy efficiency. The Orthogonal Frequency Division Multiplexing (OFDM) [1], which is the basis of several telecommunication standards such as Wi-MAX [2], long term evolution (LTE/LTE-A) [3], DVB [4], 5G [5], etc., makes it possible to satisfy most of these requests thanks to its many advantages. However, OFDM signals have strong power variations characterized by the Peak to Average Power Ratio (PAPR) [6] which generates distortions when passing through non-linear components.

The component which is at the origin of major distortions is the high power amplifier (PA) [7] especially if one seeks to increase its energy efficiency or its output power. The PA represents more than $60 \%$ of the transmitter's energy consumption [8], so its efficiency is of paramount importance. Improving the efficiency of PAs helps to reduce energy consumption in mobile equipments and base stations. It is then necessary to improve both the linearity and the energy efficiency of power amplifiers to satisfy the ever increasing need for performance, namely more services, more throughput, more users, less complexity and less consumed power.

On one hand, OFDM signals with high PAPR require a large dynamic linear amplification and on the other hand, the non-linear amplifier generates distortions and has a low energy efficiency if we operate it in its linear region. There is therefore a tradoff between linearity and efficiency at the level of the amplifier and it is difficult to satisfy mutually these two objectives.

Faced with this situation, a multitude of methods or techniques have been proposed in the literature to improve the performance of multi-carrier transmitters. A first group of methods [9], [10] consists in acting on the amplifier linearity in order to avoid distortions of the input signal. We speak, in this case, about linearization methods. A second group of methods [11], [12] focuses rather on the signal to be amplified by proposing to reduce its dynamics. This will therefore allow the amplifier to operate with better efficiency. For this second category, we talk about, PAPR reduction methods.

In this paper, two approaches will be compared: Outphasing method [13] and Iterative Dichotomy PAPR Reduction (IDP) [14] method. These two methods have a similar transmitter architecture. Two signals are first generated, then non-linearly amplified and finally combined and fed to the antenna. For the Outphasing method the two generated signals have a constant envelope (zero PAPR, non sensitive to PA) while for the IDP technique the two generated signals have low PAPR. The goal of the paper is to compare the performances of these two methods in terms of Error Vector Magnitude (EVM), BER, power efficiency and power consumption.

The originality of this paper comes from the fact that it is the first time, to the best of our knowledge, that Outphasing and IDP techniques are compared. For the same consumed power, we compare the emitted power of the two methods knowing that they have the same BER performance. Furthermore, because the two methods rely on combining synchronously the two amplified signals before the antenna, we have studied the impact of timing offset, when combining the signal after amplification.

This paper is organized as follows: In Section II a brief presentation of both Outphasing and IDP methods will be given. In section III we present the system model used for the comparison of the two techniques. Simulation results comparing the two approaches are shown in section IV. Finally, the conclusion of this work is given in section $\mathrm{V}$ 


\section{OUTPHASING AND IDP TECHNIQUES PRESENTATION}

\section{A. Waveform description}

OFDM is an orthogonal multicarrier system based on a rectangular shaping filter and the Inverse Fast Fourier Transform (IFFT). The $n^{\text {th }}$ sample of the $l^{\text {th }}$ OFDM symbol can be written:

$$
x(n)=\frac{1}{\sqrt{N}} \sum_{k=0}^{N-1} d_{k, l} e^{\frac{j 2 \pi k n}{N}}, n=0,1, \ldots, N-1
$$

where $\boldsymbol{d}_{m}=\left[d_{0, l}, d_{1, l}, \ldots, d_{N-1, l}\right]$ is the emitted vector of complex M-QAM symbols, $l$ is the index of the OFDM symbol, $k$ is the index of the subcarrier and $N$ is the total number of subcarriers.

\section{B. PA models}

Various nonlinear power amplifier models can be found in the literature. In this paper, we will use the Rapp model [15] for our analysis. The Rapp model is commonly used to model Solid State Power Amplifier (SSPA) amplifiers, its amplitudeamplitude modulation (AM/AM) transfer function is given by:

$$
f_{A M / A M}(r)=\frac{r}{\left(1+\left(\frac{r}{A_{\text {sat }}}\right)^{2 p}\right)^{\frac{1}{2 p}}}
$$

where $r$ is the instantaneous modulus of the input signal, $A_{\text {sat }}$ is the saturation voltage of the power amplifier, and $p$ is the knee factor.

The PA is operated at a given Input Back-Off (IBO) in order to avoid or at least to reduce the effects of nonlinearities. The IBO is defined, in the log scale, as follows:

$$
\mathrm{IBO}=10 \log _{10}\left(\frac{A_{\text {sat }}^{2}}{P_{r}}\right)
$$

where $P_{r}$ is the mean power of the signal at the PA input.

\section{Outphasing technique}

Outphasing has been proposed in [13]. Its main idea is to decompse the original non-constant envelope modulated signal $x(n)$ into two constant envelope signals, which are separately amplified by two highly efficient amplifiers, and then recombined and fed to the antenna. Fig.1 shows the Outphasing transmitter.

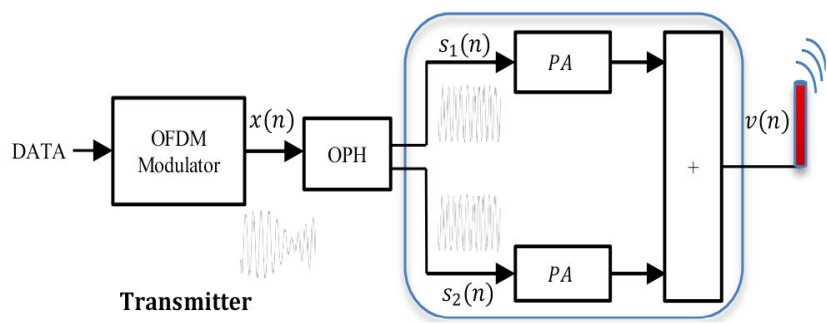

Fig. 1: Block diagram of OPH method transmitter

Fig.2 shows a geometric explanation of the construction of the two outphasing signals, the signal $x(n)$ can be decomposed into two signals $s_{1}(n)$ and $s_{2}(n)$ with constant modulus (constant envelope).

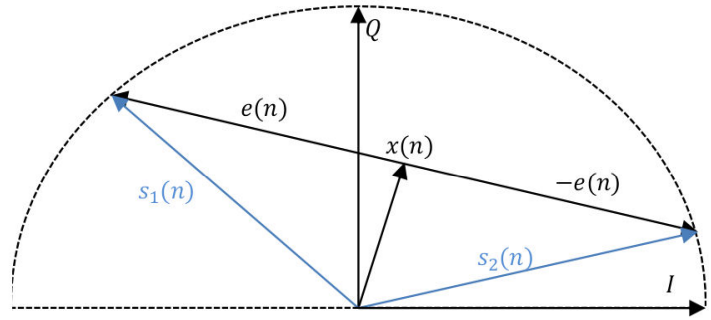

Fig. 2: Geometric explanation of the construction of the outphasing signals

A complex representation of OFDM signal $x(n)$ can be written as:

$$
x(n)=A(n) e^{j \phi(n)}
$$

where $A(n)$ is the modulus of $x(n)$ and $\phi(n)$ its phase.

The OFDM signal $x(n)$ given by equation (4) is split by the Outphasing technique into two signals with constant amplitudes, as shown in Fig. 2

$$
s_{1}(n)=x(n)+e(n)
$$

and

$$
s_{2}(n)=x(n)-e(n)
$$

where $e(n)$ is the quadrature signal of $x(n)$ and defined by

$$
e(n)=j x(n) \sqrt{\frac{A_{\max }^{2}}{A^{2}(n)}-1}
$$

where $A_{\max }$ is the maximum value of the modulus $A(n)$.

\section{IDP technique}

Iterative Dichotomy PAPR reduction (IDP) is a PAPR reduction method. It has been recently proposed in [14], its main idea is to decompose the multicarrier signal $x(n)$, with high PAPR, into $M$ signals with lower PAPR. We then use $M$ PA to amplify the $M$ signals. These signals at the output of the PA are then summed and fed to the antenna. Knowing that the $M$ signals of the decomposition have a lower PAPR, we can operate the PAs at lower IBOs and thus with a better power efficiency. In order to have similar architectures for the two transmitters, $M$ is taken equal to 2 in the following. Fig. 3 shows the IDP transmitter.

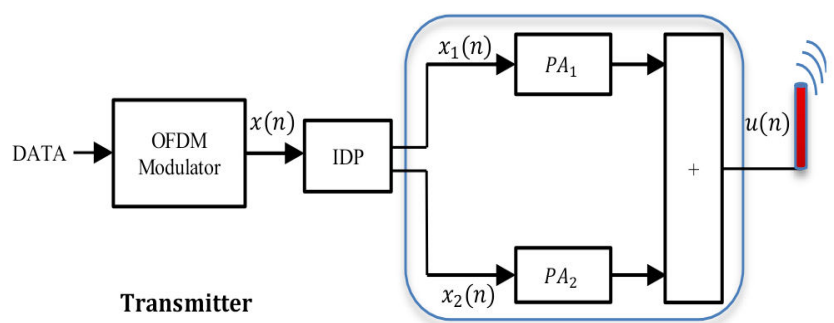

Fig. 3: Block diagram of IDP method transmitter 
The original signal $x(n)$ is decomposed into two signals: a signal with constant envelope $z_{1}(n)$ and an error signal $\varepsilon_{1}(n)$. The envelope of the first signal $x_{1}(n)$ is computed in order to minimize the power of the error signal $\varepsilon_{1}(n)$. Then, the error signal $\varepsilon_{1}(n)$ is itself decomposed into two other signals: a constant envelope signal and a second error signal and so on. At each step, we divide the error signal into two signals: a constant envelope signal and a residual error signal. The proposed IDP method is detailled in algorithm 1.

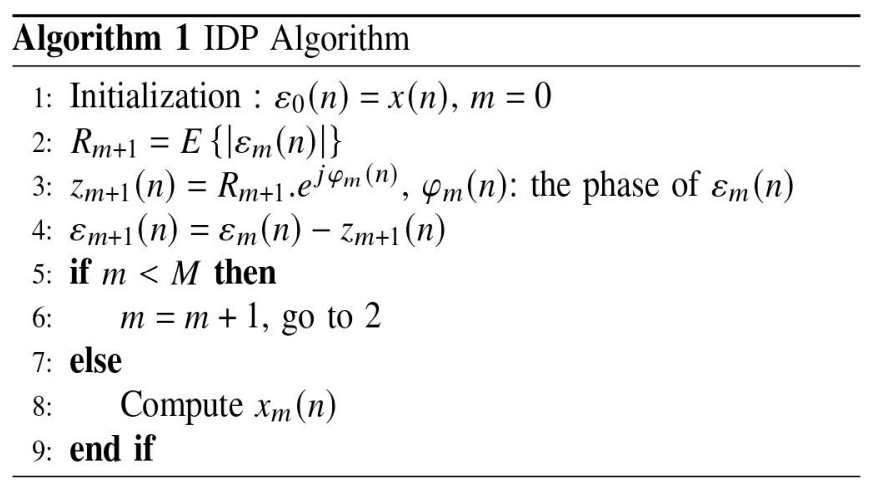

The two final signals, at the output of the IDP algorithm with $M=2$, are given by:

$$
\begin{aligned}
& x_{1}(n)=R_{1} e^{j \varphi_{x}(n)}+\frac{R_{1}}{R_{1}+R_{2}} \varepsilon_{2}(n) \\
& x_{2}(n)=R_{2} e^{j \varphi_{x}(n)}+\frac{R_{2}}{R_{1}+R_{2}} \varepsilon_{2}(n)
\end{aligned}
$$

\section{COMPARISON SYSTEM MODEL}

In this section, we will study the energy efficiency of both methods by comparing an Outphasing (OPH) based OFDM transmitter to an IDP based one.

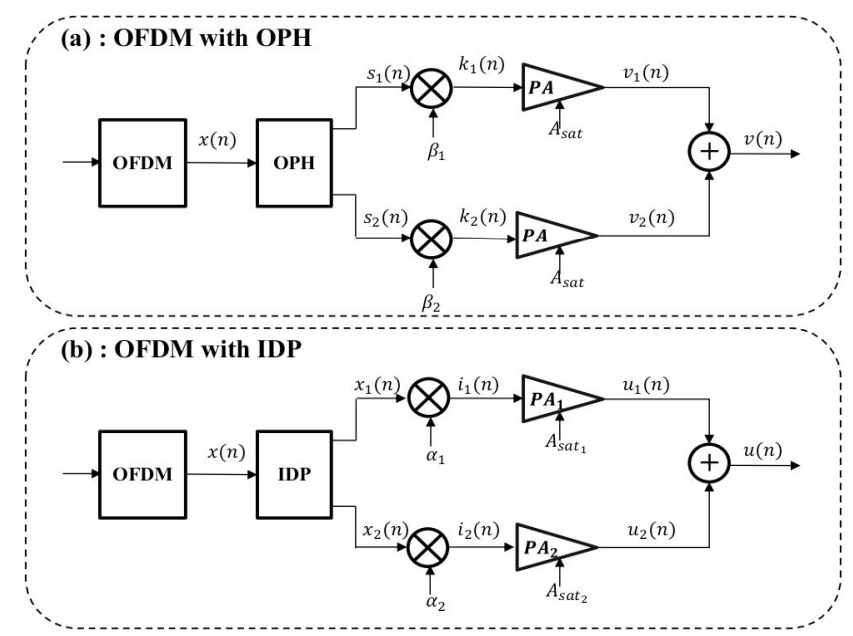

Fig. 4: OFDM transmitter (a)-Outphasing case,(b)-IDP based OFDM

Figure 4 shows the transmitters corresponding to the Outphasing scheme (Fig 4(a)) and the IDP based one (Fig 4(b)). We remind that with Outphasing method, the original signal $x(n)$ is decomposed into two correlated signals $s_{1}(n)$ and $s_{2}(n)$ with $P_{s_{1}}+P_{s_{2}}>P_{x}$, while with IDP method, the original signal $x(n)$ is decomposed into two uncorrelated signals $x_{1}(n)$ and $x_{2}(n)$ with $P_{x_{1}}+P_{x_{2}}=P_{x}$.

In order to compare the energy efficiency of the two schemes given by Fig. 4, and in order to insure fair comparison, we will compare both schemes at the same consumed power $P_{D C}$. We will assume that the consumed power $P_{D C}$, of a given PA is proportional to the saturation power. As the PA model used in the paper is the Rapp one, we will consider that the DC consumed power as given by :

$$
P_{D C}=A_{\text {sat }}^{2}
$$

In the following, we will show how to set the parameters of the two schemes in order to compare their energy efficiency. Coefficients $\alpha_{1}$ and $\alpha_{2}$ are multiplicative coefficients for adjusting the IBO of power amplifiers. In the case of Outphasing, we will operate, without loss of generality, the two PA with an IBO equal to $0 \mathrm{~dB}$ and thus, $\beta_{1}=\beta_{2}=1$.

For the amplification process, the Rapp model amplifier is used, to amplify both $k_{1}(n)$ and $k_{2}(n)$ signals (OFDM with Outphasing, Fig. 4(a)). The saturation voltage of both $P A$ used by $\mathrm{OPH}$ is $A_{\text {sat }}$, given by :

$$
A_{\text {sat }}=R
$$

where $R$ is the modulus of $s_{1}(n)$ and $s_{2}(n)$. This choice, of the saturation voltage, induces that both PA, used by the Outphasing algorithm, are operated at an IBO equal to $0 \mathrm{~dB}$.

$P A_{1}$ and $P A_{2}$ are used to amplify signals $i_{1}(n)$ and $i_{2}(n)$, in the IDP based transmitter, as presented by 4(b). The saturation voltage of $P A_{1}$ and $P A_{2}$ are $A_{s a t_{1}}$ and $A_{s a t_{2}}$ and are given by:

$$
A_{s a t_{1}}=A_{s a t} \sqrt{\frac{P_{i_{1}}}{P_{x}}} \sqrt{2}
$$

and

$$
A_{s a t_{2}}=A_{s a t} \sqrt{\frac{P_{i_{2}}}{P_{x}}} \sqrt{2}
$$

To amplify signals $k_{1}(n)$ and $k_{2}(n)$ of Outphasing method, we use the same amplifier PA with the same $A_{\text {sat }}$. While, to amplify the signals $i_{1}(n)$ and $i_{2}(n)$ we use PA with different saturation voltages. In [14] we demonstrated that the signals at the output of IDP, are uncorrelated, with respective average powers equal to:

$$
P_{x_{1}}=\frac{\pi}{4} \cdot P_{x}=0.7854 \cdot P_{x}
$$

and

$$
P_{x_{2}}=0.2146 . P_{x}
$$

Based on the assumption made in equation (10), the DC power consumed by the Outphasing based scheme is equal to: 


$$
P_{D C_{O P H}}=2 A_{\text {sat }}^{2}=2 R^{2}
$$

Regarding the IDP based scheme, the corresponding DC consumed power is equal to:

$$
P_{D C_{I D P}}=A_{\text {sat }}^{2}+A_{\text {sat }_{2}}^{2}
$$

In the next section, we will compare Outphasing and IDP techniques by considering the three following scenarios:

- Compare the output power of the two schemes: comparison with identical DC consumed power and with very low EVM (zero for outphasing) and sufficiently low for IDP so that the BER of both techniques are similar.

- Influence of a timing error combining the two amplified signals. A timing error corresponds to a phase rotation $(d \phi)$ between $k_{1}(n)$ and $k_{2}(n)$ for Outphasing and between $i_{1}(n)$ and $i_{2}(n)$ for IDP.

- Influence of a bad pairing between the two PA: we fix the knee factor of the first amplifier in both schemes to $p_{1}=2$, and for the second amplifier to $p_{2}=\gamma \cdot p_{1}$, and we compare the BER performances for various values of $\gamma$.

\section{SimUlation RESUlTS}

In this section, we compare the energy efficiency of Outphasing and IDP method. The simulation results are obtained using $10^{5}$ OFDM symbols generated using $N=1024$ subcarriers. The frequency domain data symbols were modulated using a $16-Q A M$ constellation on each subcarrier. The $P A$ used in the case of Outphasing are operated at an IBO equal to $0 d B$. The channel model used is the AWGN one.

In a first step, we compare the output power for the same DC consumption and the same BER performance. The DC power consuption for the two methods are equal: 2. $A_{\text {sat }}^{2}=A_{\text {sat }}^{2}+$ $A_{s a t_{2}}^{2}$. The parameters setting for the IDP method is done as follows: (1) we start with an IBO of the two HPA equal to zero. With such IBO, the EVM and BER are degradated because $i_{1}(n)$ and $i_{2}(n)$ have a PAPR that is low but not equal to zero. (2) We increase the IBO of the two PA. Increasing the IBO results in better performances concerning EVM and BER. (3) We stop increasing the IBO when the BER of IDP technique is similar to the one obtained by Outphasing technique. By doing so, we find that for IDP, $P A_{1}$ and $P A_{2}$ must be opareted at the same IBO of $6 d B$. With such IBO for IDP technique, we have a very small degradation of the BER compared to the linear case. The degradation is only equal to $0.1 d B$ at a $B E R=10^{-3}$

For the the same $P_{D C}$ we can have similar performances in terms of BER with $I B O(O P H)=0 \mathrm{~dB}$ and $I B O(I D P)=6 \mathrm{~dB}$. Concerning the output power (signals $v(n)$ and $u(n)$ of Fig. 4), simulation results show that the output power of IDP method is twice the one of the Outphasing method. In summary, because Outphasing has to amplify very high power signals $\left(P_{S_{1}}+\right.$ $P_{S_{2}}>>P_{x}$ ) compared to the power of the original input signal, even with higer IBO, the IDP method can reach higher output power with the same BER performance.

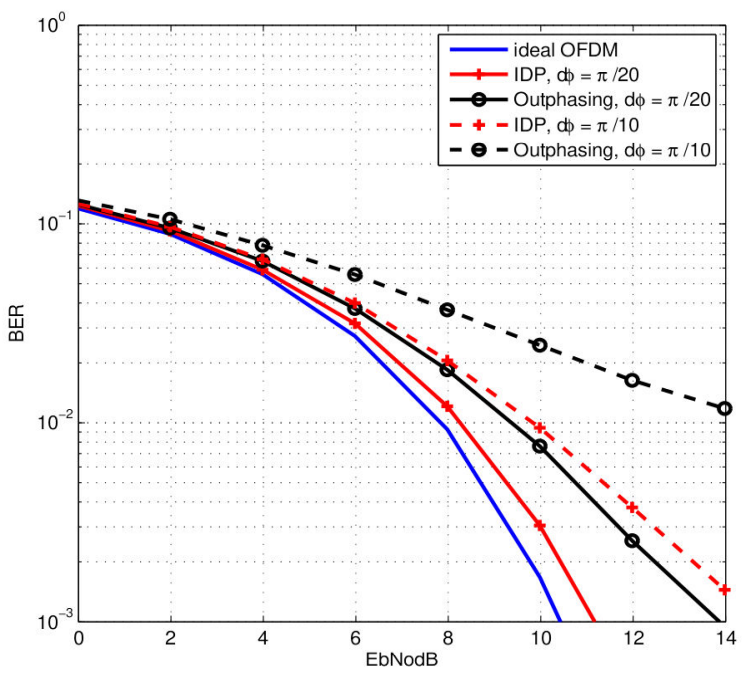

Fig. 5: BER of IDP and OPH, with timing offset $d \phi$

In the second comparison scenario, we compare the influence of timing errors on BER performances. Fig. 5 illustrates the BER performance of the OFDM signal with Outphasing and IDP methods when time missalignement $(d \phi)$ is applied between signals $k_{1}(n)$ and $k_{2}(n)$ for Outphasing and $i_{1}(n)$ and $i_{2}(n)$ for IDP. When $d \phi=\pi / 20$, we can see a small degradation of the BER between IDP and the linear case. For a BER of $10^{-3}$, there is a degradation of $0.7 d B$ for IDP and $3.5 \mathrm{~dB}$ for Outphasing compared to the ideal case. While, when $d \phi=\pi / 10$, we can notice a stronger degradation of the BER especially for the Outphasing technique. We can conclude that the IDP method is more tolerant to time misalignment than Outphasing.

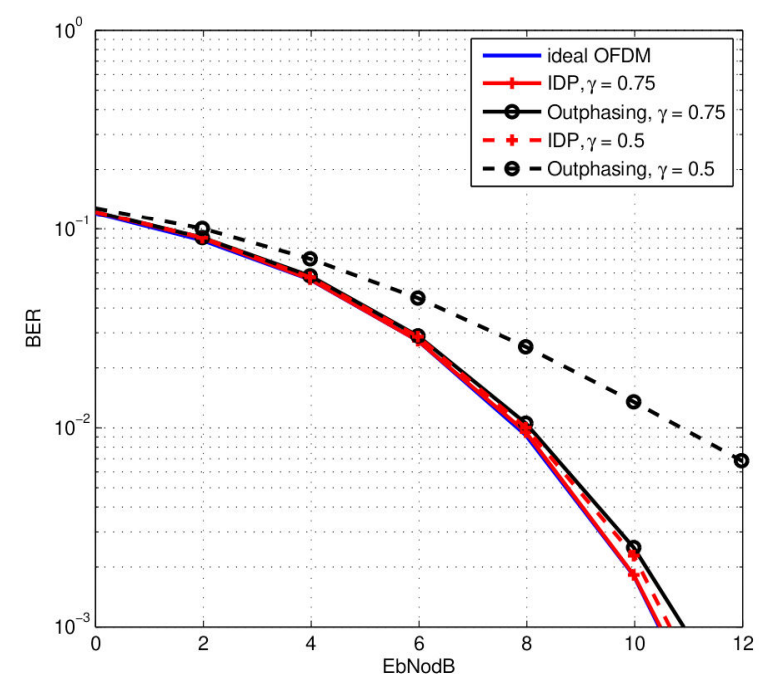

Fig. 6: BER of IDP and OPH, case of bad pairing between the two PA barnches

The last comparison is relaed to the influence of PA mismatch. Fig. 6 presents the BER performance of the two 
methods when different knee factor $p$ are used between the first and the second amplifier. For both methods : OPH and IDP, we will use $p_{1}=2$, for the PA of the first branch and $p_{2}=\gamma \times p_{1}$ for the second PA. We can see a small degradation when $\gamma=0.75$, i.e $p_{2}=1.5$. When $\gamma=0.5$, a strong degradation of Outphasing in shown, while the IDP keep the same performance.

Concerning the complexity of the two methods. From Algorithm 1, the IDP scheme requires 2 complex multiplications to compute signals $z_{1}(n)$ and $z_{2}(n)$, and it requires also 2 complex multiplications to compute the final signals $x_{1}(n)$ and $x_{2}(n)$, which gives in total $4 N$ complex multiplications. The OPH scheme requires $3 N$ complex multiplications, as one could easlily deduce form equation (7). Where, $N$ is the number of subcarriers. We can so say the for a given number of subcarriers $N, \mathrm{OPH}$ and IDP algorithms have nearly the same complexity.

\section{CONCLUSION}

In this paper we have compared the energy efficiency of two methods: Outphasing and IDP. The interest of this comparison is because both techniques have the same transmitter architecture consisting in the generation of two signals that must be amplified by two PA and then combined for transmission. The comparison has been done following the three main points: (1) the output power for the same DC consumed power and the same BER performance, (2) the influence of timing errors in the combination process and (3) the effect of a mismatch between the amplifiers. Concerning the output emitted power with same DC power and same BER performance, the IDP method outperforms the Outphasing one, with an emitted power which's twice that of OPH. Concerning timing errors, the two methods are quite impacted. However, the IDP technique seems to be more tolerant that OPH. Finally, with PA mismatch between the two branches, the IDP method shows low degradations compared to the Outphasing one.

\section{REFERENCES}

[1] S.-Y. Lien, S.-L. Shieh, Y. Huang, B. Su, Y.-L. Hsu, and H.-Y. Wei, "5G new radio: Waveform, frame structure, multiple access, and initial access," IEEE communications magazine, vol. 55, no. 6, pp. 64-71, 2017.

[2] 3rd Generation Partnership Project; Technical Specification Group Services and System Aspects; Architecture Enhancements for Non-3GPP Accesses (Release 10, 3GPP Standard TS 23.402 V10.7.0, Mar. 2012.

[3] Evolved Universal Terrestrial Radio Access (E-UTRA); User Equipment (UE) Radio Transmission and Reception (Release 10), Version 10.3.0, 3GPP Standard TS 36.101, Jun. 2011.

[4] Digital Video Broadcasting (DVB): Framing Structure, Channel Coding and Modulation for Digital Terrestrial Television Broadcasting System(DVB-T2), v1.2.1, ETSI Standard 302755, 2010.

[5] 3rd Generation Partnership Project; Technical Specification Group Radio Access Network; NR; Physical Layer; General Description (Release 15), "V1.0.0," document, vol. TS, no. 38, p. 201, 3GPP 2017.

[6] B. Xu, P. Almers, J. Chen, and J. Chen, "Method and device for Peak to- Average Power Ratio Reduction in an OFDM system," May 2018 US Patent App. 15/861,196.

[7] E. Costa, M. Midrio, and S. Pupolin, "Impact of amplifier nonlinearities on OFDM transmission system performance", IEEE Communications Letters, vol. 3, no. 2, pp. 37-39, 1999.

[8] H. Bogucka and A. Conti, "Degrees of freedom for energy savings in practical adaptive wireless systems," IEEE Communications Magazine, vol. 49, no. 6, 2011, pp. 38-45.

[9] K. Chuang, "A Perspective on Linearization and Digital Pre-Distortion for Wireless Radio Systems," 2020 IEEE Topical Conference on RF/Microwave Power Amplifiers for Radio and Wireless Applications (PAWR), San Antonio, TX, USA, 2020, pp. 38-41,

[10] R. Zayani, H. Shaïek and D. Roviras, "Ping-Pong Joint Optimization of PAPR Reduction and HPA Linearization in OFDM Systems," in IEEE Transactions on Broadcasting, vol. 65, no. 2, pp. 308-315, June 2019.

[11] H. Merah, M. Mesri, and L. Talbi. "Complexity reduction of PTS technique to reduce PAPR of OFDM signal used in a wireless communication system.” IET Communications vol. 13, no. 7 2019. pp. 939946

[12] M. Mesri, K. Tahkoubit, H. Merrah, and A. Ali-pacha. "Partial Transition Sequence Algorithms for Reducing Peak to Average Power Ratio in the Next Generation Wireless Communications Systems." Journal of Electrical Systems vol. 13, no. 1. 2017.

[13] Zhang, Xuejun, Lawrence E. Larson, and Peter Asbeck. Design of linear RF outphasing power amplifiers. Artech House, 2003.

[14] K. Tahkoubit, A. Ali-Pacha, H. Shaiek, and D. Roviras, "Iterative Dichotomy PAPR Reduction Method for Multicarrier Waveforms", IEEE Communications Letters, vol. 23, no. 11, pp. 2073-2076, 2019.

[15] M. C. P. Paredes, F. Grijalva, J. Carvajal-Rodriguez and F. Sarzosa, "Performance analysis of the effects caused by PA models on an OFDM signal with high PAPR", IEEE Second Ecuador Technical Chapters Meeting (ETCM), pp. 1-5. IEEE, 2017. 